\title{
Neutron diagnostics at the Wendelstein 7-X stellarator
}

\author{
W. Schneider ${ }^{a *}$, B. Wiegel ${ }^{b}$, F. Grünauer ${ }^{c}$, R. Burhenn $^{a}$, S. Koch ${ }^{b}$, \\ H. Schuhmacher ${ }^{b}$ and A. Zimbal ${ }^{b}$ \\ ${ }^{a}$ Max-Planck-Institut für Plasmaphysik, EURATOM Association, Teilinstitut Greifswald, \\ Wendelsteinstraße 117491 Greifswald, Germany, \\ E-mail: wolfgang.schneider@ipp.mpg.de \\ ${ }^{b}$ Physikalisch-Technische Bundesanstalt, \\ Bundesallee 100, 38116 Braunschweig, Germany \\ ${ }^{c}$ Physics Consulting, \\ Herzog-Otto-Weg 17, 85604 Zorneding, Germany
}

\begin{abstract}
The stellarator W7-X, presently under construction at the Institute for Plasma Physics in Greifswald, will be equipped with a set of neutron monitors in order to study the time behaviour of neutron emission generated during D-D plasma operation and neutral beam heating with Deuterium. Each of these neutron monitors consists of several neutron detector tubes inserted in a dedicated moderator. The neutron monitors at W7-X are designed to monitor neutron yields with a time resolution of $5 \mathrm{~ms}$ and with a statistical uncertainty of better than $15 \%$. One of the monitors is located in the centre above the stellarator. The other five monitors are distributed around the torus. A prerequisite for the determination of the absolute neutron source strength produced by $\mathrm{D}(\mathrm{d}, \mathrm{n})^{3} \mathrm{He}$ fusion reactions in the plasma is an in-situ calibration with a neutron source of known source strength. During such a calibration procedure, the neutron source will be moved along the torus axis and the count rates of the different neutron monitors will be measured. In a first benchmark experiment, an ${ }^{241} \mathrm{AmBe}$ neutron source was moved along the torus axis within one module of the stellarator and the neutron signals were measured by a De Pangher Long Counter outside of the cryostat chamber as a function of the neutron source position. These measurements have been compared with predictions of Monte Carlo calculations (MCNP) of the neutron propagation from the location of the neutron source to the long counter. The concept of neutron monitors will be reported together with results from the benchmark experiment and results from MCNP calculations. The neutron monitor system is the first part of several neutron diagnostic systems such as neutron activation system, neutron profile camera planned for future neutron analysis. A short survey of these neutron diagnostic tools of W7-X will be given.
\end{abstract}

KEYWORDS: Nuclear instruments and methods for hot plasma diagnostics; Detector modelling and simulations; Detector alignment and calibration methods.

* Corresponding author. 


\section{Contents}

$\begin{array}{ll}\text { 1. Introdution } & 1\end{array}$

2. Objectives and concept of neutron monitors 2

2.1. Objectives 2

2.2. Concept of neutron monitors 2

3. Calibration of neutron monitors 3

4. MCNP model of W7-X 4

5. Test calibration at W7-X module no. 5

5.1. Experimental setup at W7-X 6

$\begin{array}{ll}\text { 5.1.1.Track system for moving the neutron source } & 6\end{array}$

5.1.2. MCNP model of the De Pangher Long Counter 6

5.1.3. The PTB Long Counter at W7-X

5.1.4. Calibration source at W7-X $\quad 8$

5.2. Performing the experiment at W7-X 9

6. First experimental results and comparison with calculations $\quad 10$

6.1. Long counter data as function of time 10

6.2. Long counter data as function of path length 11

6.3. Comparison of measured data with MCNP calculations 12

6.4. Technical conclusions from neutron experiment at module $5 \quad 15$

7. Set of complementary neutron diagnostics at W7-X 15

$\begin{array}{ll}\text { 8. Summary } & 16\end{array}$

\section{Introduction}

Wendelstein 7-X is a drift-optimised stellarator with improved thermal and fast ion confinement and with the aim to demonstrate the possibility of a quasi steady-state operation under reactor relevant plasma conditions (30 min discharge duration, ion temperature $T_{\mathrm{i}}$ up to $10 \mathrm{keV}$ and ion density $n \geq 10^{20} \mathrm{~m}^{-3}$ ) [1]. W7-X is equipped with 70 superconducting coils in order to generate a specific twisted magnetic confinement and to realise a magnetic field strength of up to $3 \mathrm{~T}$ on the magnetic axis. Its plasma volume is about $30 \mathrm{~m}^{3}$ with a major radius of $5.5 \mathrm{~m}$ and a minor radius of about $0.5 \mathrm{~m}$. The heating of the W7-X plasma will be done by ECRH (electron cyclotron resonance heating) with gyrotrons at $140 \mathrm{GHz}$, ICRH (ion cyclotron resonance heating) and NBI (neutral beam injection) up to a total power of $30 \mathrm{MW}$ in the final stage. A detailed survey of machine properties and features of diagnostics planned for W7-X is given in [2]. 


\section{Objectives and concept of neutron monitors}

\subsection{Objectives}

The objective of neutron monitors of W7-X is to study the time behaviour of the neutron emission generated during D-D plasma operation and during neutral beam heating with Deuterium. The neutron rate represents a direct measure of the fusion rate and fusion energy and provides information on the ion temperature.

In order to measure the neutron flux at several places around the stellarator neutron monitors are required, which consist of different detectors and specific shaped moderators to cover a wide range of neutron energy and intensity. Depending on different heating scenarios neutron emission rates in the range from $10^{11} \mathrm{~s}^{-1}$ to $10^{16} \mathrm{~s}^{-1}$ have to be expected. The analysis of measured neutron signals in and around the torus and its interpretation requires the application of MCNP modelling. Neutron measurements and the comparison with MCNP simulations have already been described in [3] for the precursor of W7-X, the stellarator W7AS. Generally, there exist only a few published results of MCNP aided modelling for stellarators compared to the large number of publications of MCNP results for tokamaks.

\subsection{Concept of neutron monitors}

In order to obtain information on the total neutron emission rate with a relative uncertainty of $15 \%$ or better and with a time resolution of $5 \mathrm{~ms}$ a set of 5 neutron monitors will be distributed around the torus and one central neutron monitor will be located in the centre of the stellarator above of the W7-X cryostat. These six neutron monitors have to fulfil both the requirements of authorities regarding radiation protection and will also be used as diagnostic instrument for the investigation of the plasma. As a consequence of these tasks two types of neutron monitors are in development for its deployment at W7-X.

The function of the central neutron monitor is to detect neutrons from the full space of a hemisphere as a kind of reference system in order to document the annual generation of neutrons with respect to the neutron budget permitted by the authorities for radiation protection. This neutron monitor will be located $3.9 \mathrm{~m}$ above the equatorial plane and is designed to exhibit a large opening angle of about $+90^{\circ}$. It will be equipped with up to five detectors (for instance: two ${ }^{3} \mathrm{He}$-counting tubes, one $\mathrm{BF}_{3}$-counting tube and two U-fission chambers) to afford the full sensitivity and dynamic range.

The outer neutron monitors will be placed every $72^{\circ}$ of the torus circumference at a distance of $0.9 \mathrm{~m}$ from the outer vessel at a height of $1.5 \mathrm{~m}$ above the equatorial plane. Each neutron monitor of this type has to measure predominantly the neutrons coming from a fifth part of the torus. These five monitors are designed to exhibit a directional dependent sensitivity with a reduced opening angle of about $\pm 45^{\circ}$ and are directed towards the plasma axis. The expected neutron flux at these positions varies from $5 \cdot 10^{3} \mathrm{~cm}^{-2} \mathrm{~s}^{-1}$ to $5 \cdot 10^{8} \mathrm{~cm}^{-2} \mathrm{~s}^{-1}$ depending on the plasma scenario. The arrangement of the six neutron monitors around the stellarator W7-X is shown in figure 1.

The various possible plasma conditions at $\mathrm{W} 7-\mathrm{X}$ require the consideration of an adequate dynamic and sensitivity range, a specific energy dependence of the monitor and a particular angle dependence. It was considered to be important for the response function of the neutron monitor to achieve a flat sensitivity behaviour with respect to the neutron energy. 


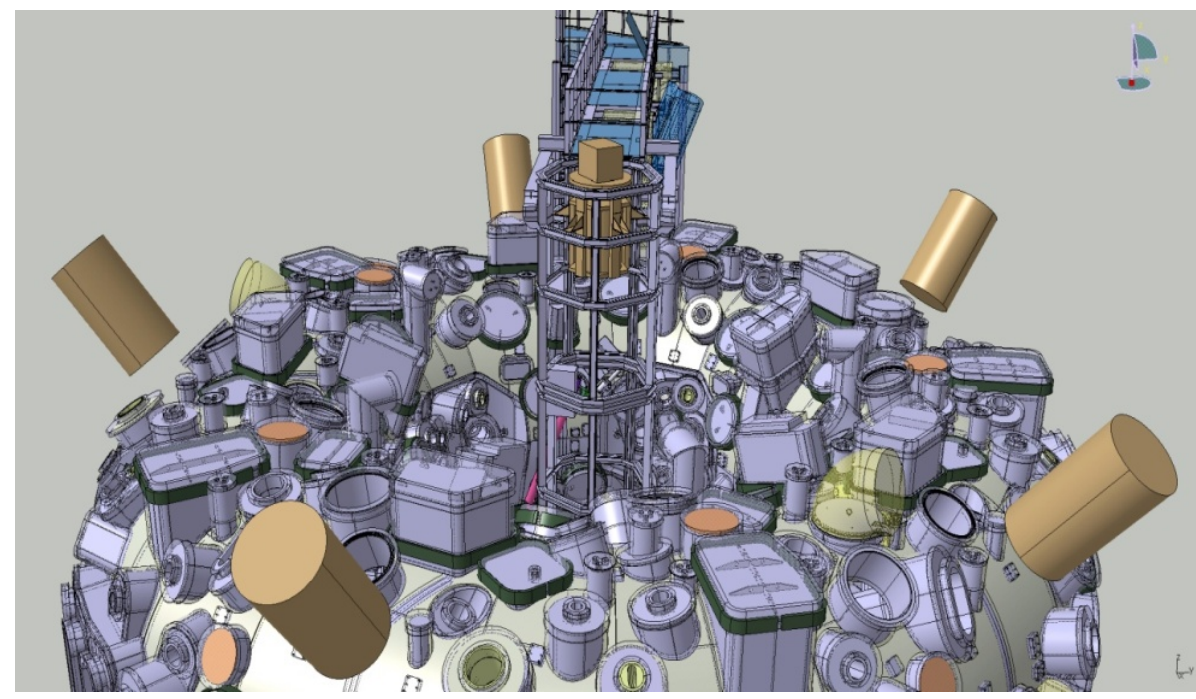

Figure 1. Sketch of the upper part of W7-X with the positions of the five outer monitors and the central neutron monitor located within the support structure of the Thomson diagnostics.

\section{Calibration of neutron monitors}

In order to determine the absolute neutron emission rate produced by $\mathrm{D}(\mathrm{d}, \mathrm{n})^{3} \mathrm{He}$ fusion reactions in the plasma, one of the first tasks is to perform an in-situ calibration of the six peripheral neutron monitors with a neutron source of known source strength. The procedure of such a calibration can be performed in two ways. One possibility is to locate the neutron source at fixed positions along the track which approximates the torus axis and measure the neutrons arriving at each monitor. The other way, which is foreseen for W7-X calibration of neutron monitors, consists in a slow continuous movement of the neutron source along the full torus axis and the time dependent registration of neutron signals in all neutron monitors of W7-X. In a second step one has to conclude from the situation of the line source, realized by the moving source, to an expanded 3D plasma.

The aim is to design each outer neutron monitor suchlike that it mainly detects neutrons generated in a certain part of the plasma volume of W7-X. Since there will be no sharp limits also neutrons from other parts of the plasma will be detected but with much lower efficiency. A correct weighing of the contributions from the five outer monitors and the central monitor is mandatory. In order to be as independent on changes in the material composition in the vicinity of the monitors a constant neutron energy response of each monitor should be achieved.

The neutron monitors detect the neutrons through the wall of the plasma vessel with a thickness of $17 \mathrm{~mm}$ stainless steel and through an outer vessel wall with a thickness of $25 \mathrm{~mm}$ stainless steel. In addition, further components such as the superconducting coils or pipelines for different media can cross the way of neutrons from the source to the neutron monitor. Because of the geometry of an advanced stellarator like W7-X with its twisted 3-dimensional magnetic field coils the setup for the calibration equipment is more complex compared to the situation in a tokamak. The torus axis of W7-X differs in its $X$-, $Y$ - and $Z$-values up to about $50 \mathrm{~cm}$ compared to a circular axis in a tokamak. To give an example complex geometry of W7-X 
figure 2 shows a vertical and a horizontal cross section of module 5, which was used for a test calibration.
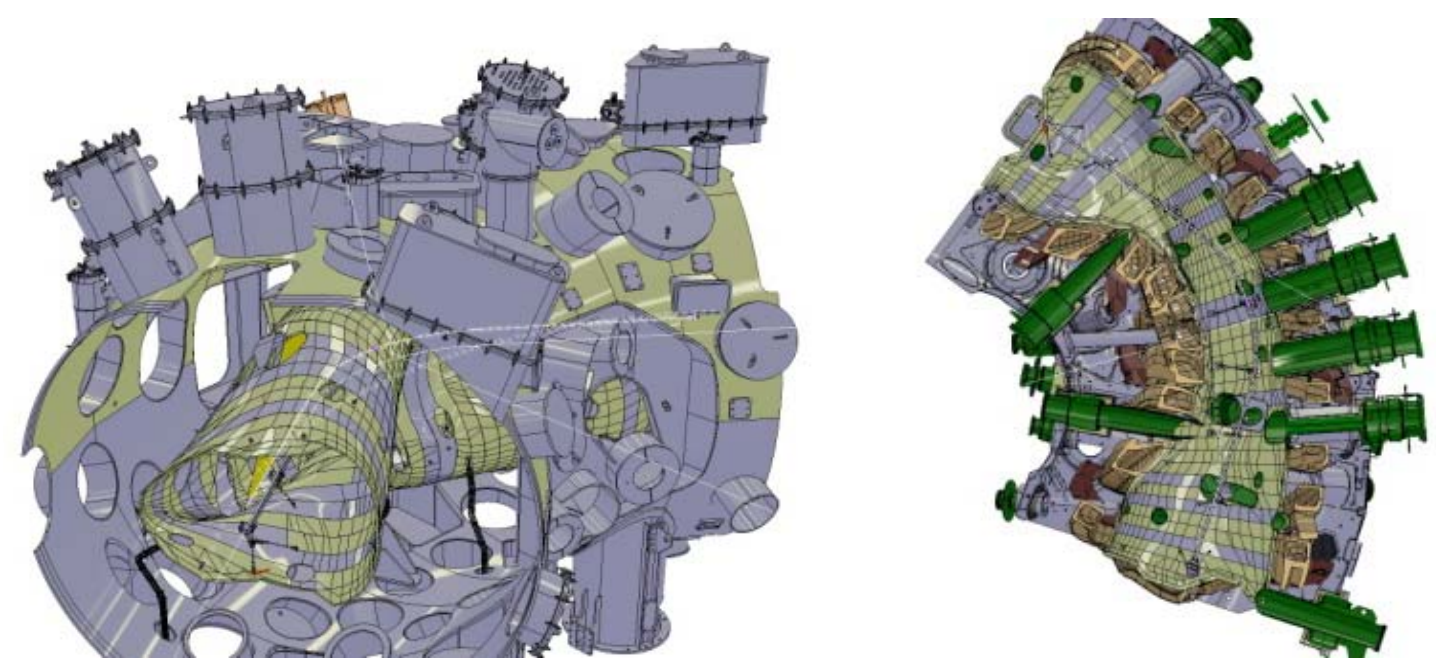

Figure 2. Vertical (left) and horizontal (right) cross section of module 5. The shape of the inner vessel gives an impression of the complex 3-dimensional plasma of W7-X. The sketches show parts of the rail track system used during the calibration experiment performed in August 2011 which is described in detail in the following sections.

\section{MCNP model of W7-X}

During the measurement with a neutron source in August 2011 the environment inside and outside the plasma vessel was not exactly the same compared to the situation of the fully equipped stellarator. Some "structure" materials as e.g. water of the coolant system did not yet exist during the measurement. The influence of these additional components on the neutron count rates in the future calibration experiments during the commissioning phase of W7-X has to be known for correct analysis of the fusion rate. On the other hand, repeating the measurement for each change of the setup would be a huge effort.

Therefore, a Monte Carlo model of the plasma vessel was created to study the influences of all structure materials and its sensitivity to all changes in the environment. MCNP5 [4] was used as Monte Carlo neutron transport code. However, the geometry of the real plasma vessel is quite complex. In the Monte Carlo model some approximations had to be made. In the model the plasma vessel is a torus which is not the case in reality. The model includes ports in the plasma vessel that have all the same geometry and are arranged symmetrically around the plasma vessel in the model, which is also not the case in reality. For simplicity, the coils around the plasma vessel are approximated by rings.

Figure 3 shows a 3-dimensional picture of the W7-X model generated with MCAM [5]. 


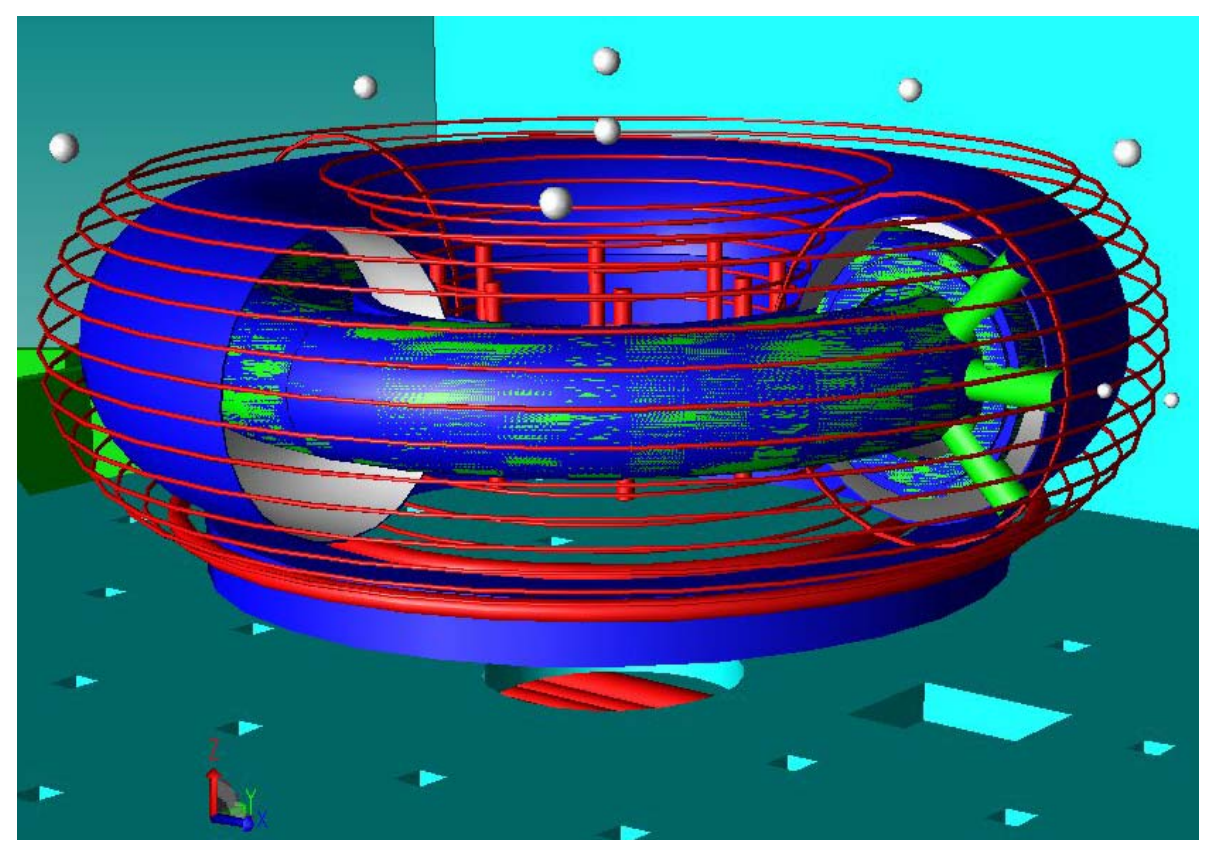

Figure 3. 3-dimensional picture of the MCNP W7-X model generated with MCAM [5]. The outer blue torus is the outer stainless steel vessel which is "opened" for one module. This gives free sight to the inner vessel and some ports (bright green). The red rings and cylinders represent the magnet coils and water pipes, respectively. The light grey spheres give possible positions of the neutron monitors (large spheres) and are used for tallying neutron spectra (large and small spheres).

\section{Test calibration at W7-X module no. 5}

Due to the complexity of the geometry of the stellarator W7-X and its simulation with MCNP it was planned to perform a test of the W7-X Monte Carlo model as long as not all modules were physically installed. During the assembly period, in which four out of five modules were installed, an easy access into the inner vessel was still possible.

In this first benchmark experiment an ${ }^{241} \mathrm{AmBe}$ neutron source with known source strength was moved along the torus axis within one module of the stellarator (e.g. along the fifth part of the torus axis) and the neutron signals were measured outside of the cryostat chamber in dependence on the position of the neutron source by a De Pangher Long Counter (LC) available at PTB. It should be mentioned that the position of the LC used in this experiment does not correspond to one of the nominal positions of the outer neutron monitors at W7-X. Considering the assembly situation during the experiment we decided to locate the LC near the middle of module 5. The nominal positions of the outer neutron monitors at W7-X will be over the section lines between the modules.

In the following we describe the track system for moving the neutron source, the LC and the setup of the experiment. 


\subsection{Experimental setup at W7-X}

\subsubsection{Track system for moving the neutron source}

A single-track system with a battery-driven radio-controlled cart was constructed in such a way that the path on which the neutron source moves is the projection of the mean plasma centre onto the equatorial plane at $Z=0 \mathrm{~mm}$. Figure 4 shows on the left hand side the track structure as it was designed with a CAD program (CATIA [6]) and on the right hand side the track system during installation.
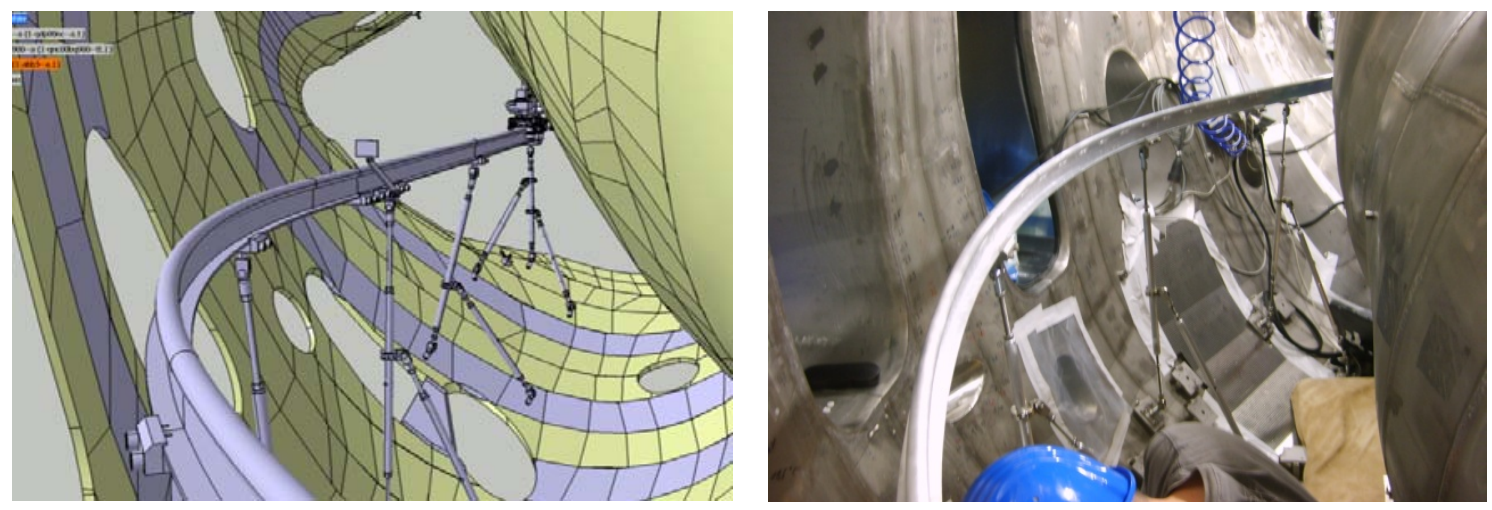

Figure 4. Single-track system for moving the neutron source along a fixed path. Left: view in CATIA model, right: view during the installation.

The cart has two vertical stands in order to fix the ${ }^{241} \mathrm{AmBe}$ neutron source at three different heights. With this the Z-coordinates of the centre of the neutron source are fixed to $Z_{\text {src }}=-20 \mathrm{~mm},+100 \mathrm{~mm}$ and $+300 \mathrm{~mm}$. To determine the position of the source in the $X-Y$ plane the path length $s$ along the cart track was used and the count rate of the LC was recorded as a function of $s$. Labelled tags were fixed on the track structure every $250 \mathrm{~mm}$ and at some additional points of interest. A W-LAN camera which was mounted on the cart permanently transmitted a picture showing the track line and the labelled tags.

\subsubsection{MCNP model of the De Pangher Long Counter}

For the test calibration experiment we used a well characterized De Pangher Long Counter which is routinely used at the PTB irradiation facility with radionuclide sources. A nearly identical long counter of NPL was characterized numerically [7] and experimentally compared to the PTB instrument [8]. The original MCNP file of the NPL De Pangher Long Counter [9] was simplified in such a way that the model is still adequate to describe the PTB LC and included into the model of W7-X.

To demonstrate that our MCNP model is a good description of the real PTB LC the count rates of the direct contribution of a ${ }^{252} \mathrm{Cf}$ source at a distance of $170 \mathrm{~cm}$ were measured in the PTB bunker room facility as a function of rotation angle (vertical rotation axis through the centre of the LC). Figure 5 shows the measured count rates (relative to $0^{\circ}$ ) and the calculated response of the long counter. The agreement between measurement and calculation is better than $4 \%$ for angles up to $90^{\circ}$ and up to $10 \%$ for larger angles. 


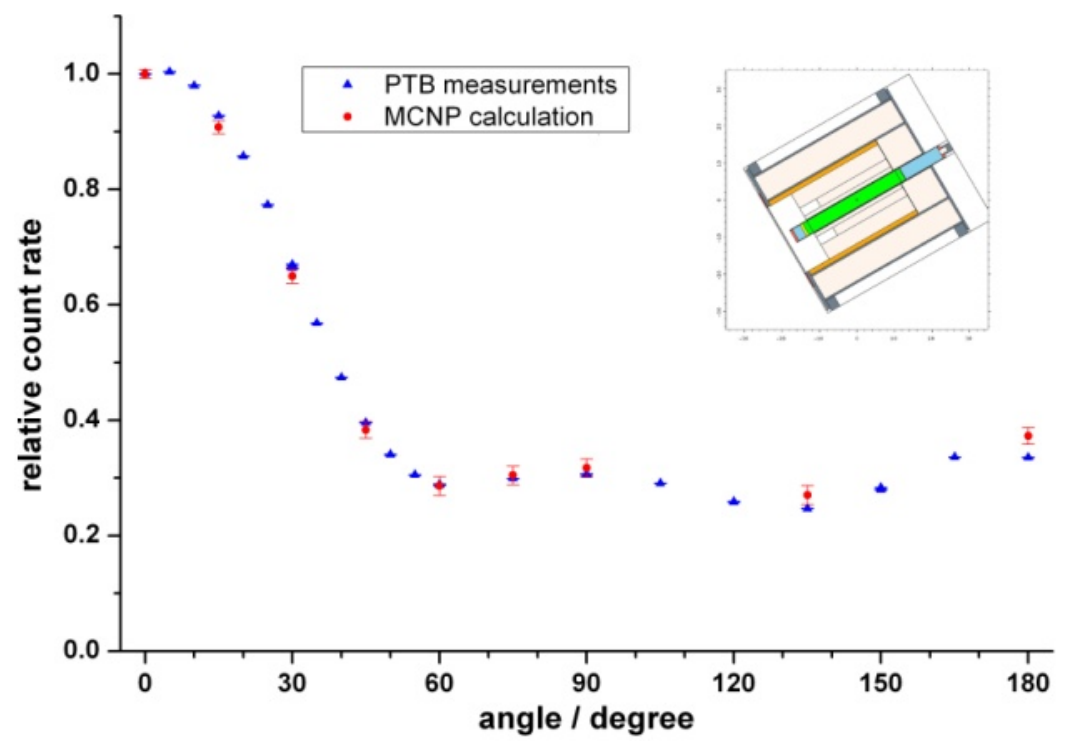

Figure 5. Comparison of calculated (MCNP) and measured count rates of the PTB De Pangher Long Counter as function of rotation angle.

\subsubsection{The PTB Long Counter at W7-X}

In the following we will refer to the reference point or origin of the long counter as $O_{\mathrm{LC}}$ which is the origin in the MCNP LC model and it is used to define the position of the LC in the coordinate system of the experimental hall. This allows to calculate the distances between the $\mathrm{LC}$ and the neutron source. The point $O_{\mathrm{LC}}$ is located on the cylinder axis of the long counter in the plane of the polyethylene front plane which is $73 \mathrm{~mm}$ behind the front plane of the Cd cover.
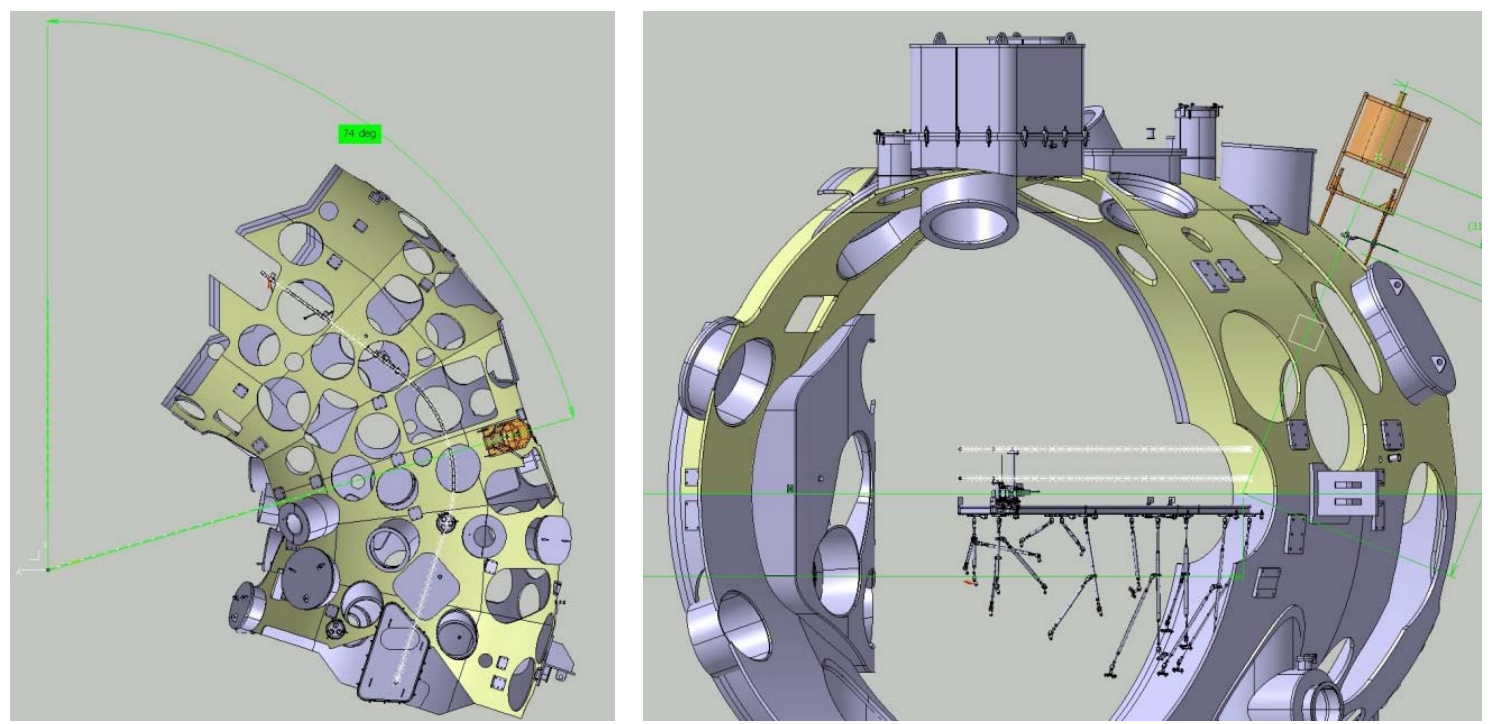

Figure 6. Position of PTB long counter at W7-X module 5. Left: top view; right: side view. 
The LC was mounted $2.26 \mathrm{~m}$ above the equatorial plane of W7-X ( $Z=0 \mathrm{~mm})$, see left hand side of figure 6 . The extension of the cylinder axis of the LC crosses the track line at path length $s=-201 \mathrm{~mm}$. For this coordinate the distance from the reference point $O_{\mathrm{LC}}$ to the centre of the neutron source at $Z_{\mathrm{src}}=300 \mathrm{~mm}$ is $2145 \mathrm{~mm}$. The angle between the cylinder axis and the equatorial plane is $68^{\circ}$, see right hand side of figure 6 .

Figure 7 shows the position of the LC as it was positioned during the test calibration experiment without the inner and outer vessel and only the non-planar superconducting coils. The light green surface indicates the approximate detection range of the LC limited below by the track of the neutron source.

The data acquisition for the LC was realized with conventional analogue NIM electronic modules. The high voltage power supply and the pre-amplifier were located nearby the LC. The main amplifier and the other components of the data acquisition system were placed more than $50 \mathrm{~m}$ away. The amplified counter signal was converted to a TTL signal and counted by a data logger [10]. In parallel, the amplified signal was recorded and stored as pulse height spectrum. The latter serves only as a check of the stability of the LC signals during the whole measuring campaign.

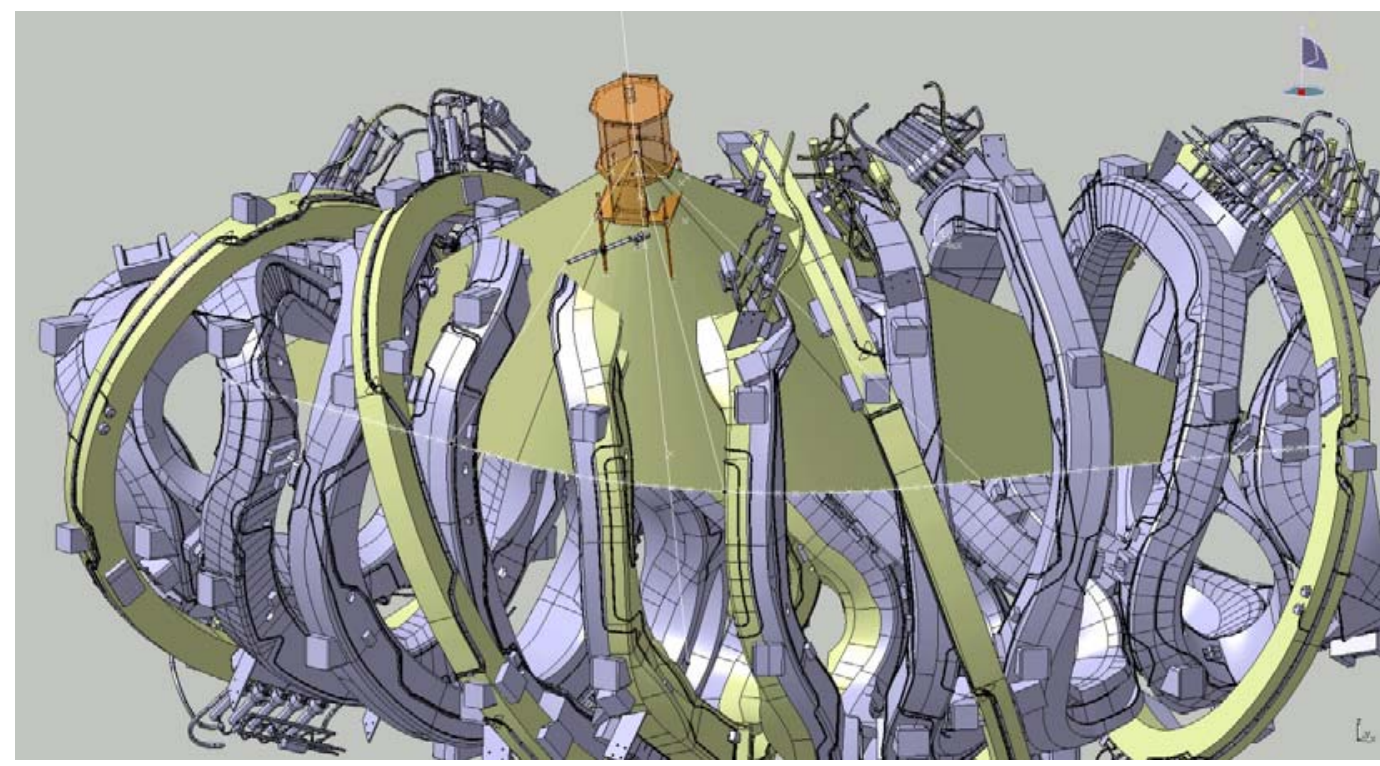

Figure 7. View of the detection range of the PTB LC through the coil system of module 5.

\subsubsection{Calibration source at W7-X}

The procedure to perform the calibration of the neutron monitors is to use a neutron source of known source strength and to measure the count rates in the neutron monitors as function of source position to simulate the extended plasma volume (see sec. 3).

In the ideal case, the neutron spectrum emitted by the source should be as close as possible to the spectrum of neutrons emitted during the fusion process. In figure 8 (right), the expected neutron spectrum for a D-D fusion plasma with different heating schemes is shown [11]. This spectrum was determined with a neutron spectrometer developed at PTB at the Joint European Torus (JET). On the left side of figure 8, the neutron spectra of typically available neutron 
sources are shown. The spectrum which fits the expected fusion neutron spectrum best is produced by ${ }^{241} \mathrm{AmB}$ sources, or other $(\alpha, \mathrm{n})$ neutron sources based on Boron. The drawback of ${ }^{241} \mathrm{AmB}$ is that these sources have typically very low emission rates and are not produced any more at the moment. ${ }^{252} \mathrm{Cf}$ sources are available with very high emission rates but their spectrum differs very much from the expected one. ${ }^{241} \mathrm{AmBe}$ sources are a reasonable compromise as they are available with sufficient source strength for reasonable prices and their neutron spectrum covers the whole energy range up to $11 \mathrm{MeV}$. In the MCNP simulation, the prediction of the monitor count rates is based on the actual neutron spectrum emitted by the source. Later, when applying this calibration to plasma emitted fusion neutrons, the difference in the neutron spectrum during the calibration and the operation has to be accounted for by an MCNP simulation using the expected fusion neutron spectrum.
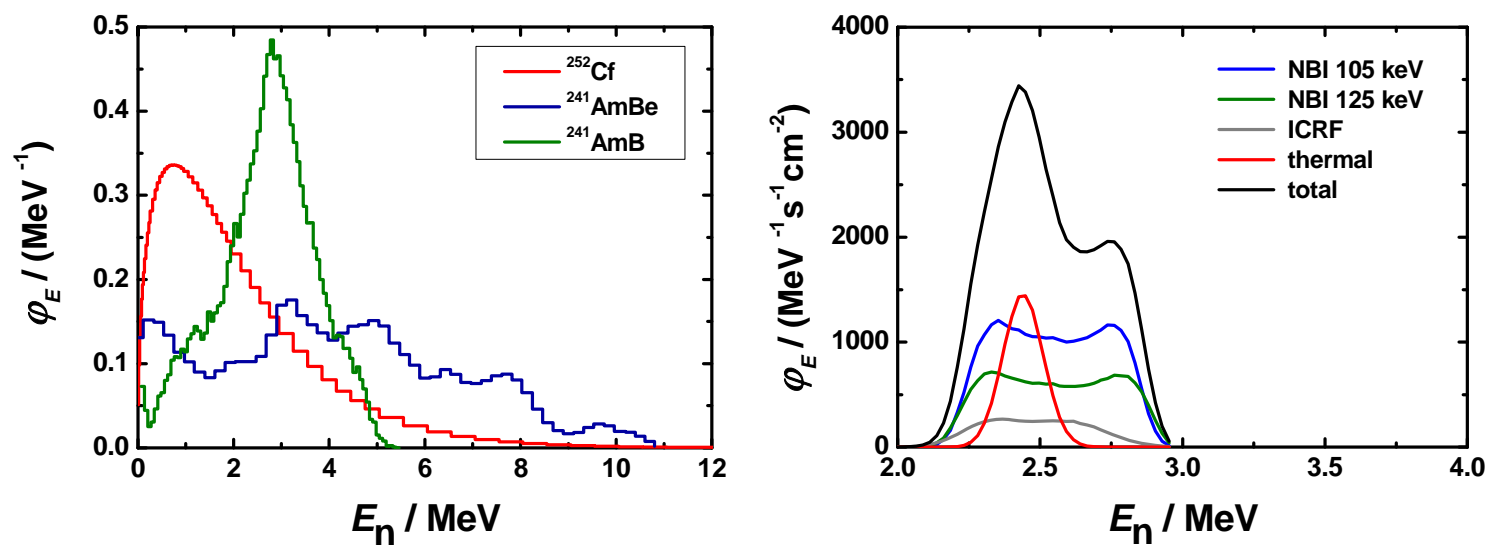

Figure 8. Left: Neutron spectra of typically available neutron sources. The spectra are normalized to unit neutron fluence [12, 13, 14]. Right: Expected neutron spectrum for a D-D fusion plasma with different heating schemes [11].

Before the LC was shipped from PTB to Greifswald the efficiency was determined with a ${ }^{241} \mathrm{AmBe}$ source of known source strength at a distance of $55 \mathrm{~cm}$ from $O_{\mathrm{LC}}$. Later, at IPP Greifswald, the same setup was used to determine the source strength of a ${ }^{241} \mathrm{AmBe}$ source which was bought by IPP shortly before the experiment. The neutron source strength as provided by the source supplier was $(1.1 \pm 0.2) \cdot 10^{6} \mathrm{~s}^{-1}$. Using the efficiency factor obtained at PTB, the source strength was determined to be $B_{\mathrm{AmBe}-\mathrm{IPP}}=(1.49 \pm 0.10) \cdot 10^{6} \mathrm{~s}^{-1}$ which is about $35 \%$ larger than the nominal value. This value of the measured source strength was used for all MCNP calculations presented in this paper. The experimental conditions at PTB and IPP of course differ with respect to neutrons scattered by the different environments. Therefore, a direct comparison of the IPP ${ }^{241}$ AmBe neutron source to the PTB standard source is planned.

\subsection{Performing the experiment at W7-X}

The experimental campaign was performed at IPP Greifswald from August $5^{\text {th }}$ to $7^{\text {th }} 2011$. In the main part of the experiment, the neutron source was mounted on the battery-driven cart. Start, stop and cart velocity were controlled from outside the vessel via a radio-control-unit. Several scans with three different vertical positions of the source with respect to the equatorial plane have been done at $Z_{\text {src }}=-20 \mathrm{~mm}, 100 \mathrm{~mm}$ and $300 \mathrm{~mm}$, respectively. The total measuring 
time of the campaign was 33 hours. The determination of the source strength of the IPP ${ }^{241}$ AmBe neutron source was performed two times, before and after the main part of the experiment to check whether any parameter of the data acquisition system had changed.

For all measurements in this experiment the time reference was taken from a standard radio-controlled watch to which all computers and the logger were synchronized once. The sampling interval of the data logger unit was set to $5 \mathrm{~s}$ for all measurements.

\section{First experimental results and comparison with calculation}

\subsection{Long counter data as function of time}

The most direct method to visualise the measured data is to sum up all intervals of the data logger (each $5 \mathrm{~s}$ ) $N_{i}$ between two time stamps $t_{i-1}$ and $t_{i}$. Figure 9 shows the count rates $n(t)=N_{i} /\left(t_{i}-t_{i-1}\right)$ as a function of time $t$ (red histogram, left ordinate) for a continuous scan of six hours. The corresponding source position $s_{\text {src }}$ is shown as upper abscissa. The source velocity $v_{\text {src }}(t)=\left(s_{i}-s_{i-1}\right) /\left(t_{i}-t_{i-1}\right)$ is shown as blue histogram corresponding to the right ordinate. When the cart reaches an end switch, the motor of the cart changes its rotation sense and the cart moves in the opposite direction. Hence the velocity changes its sign. Since the time stamp is randomly distributed within the $5 \mathrm{~s}$ interval on the data logger, the velocity scatters around an average value.

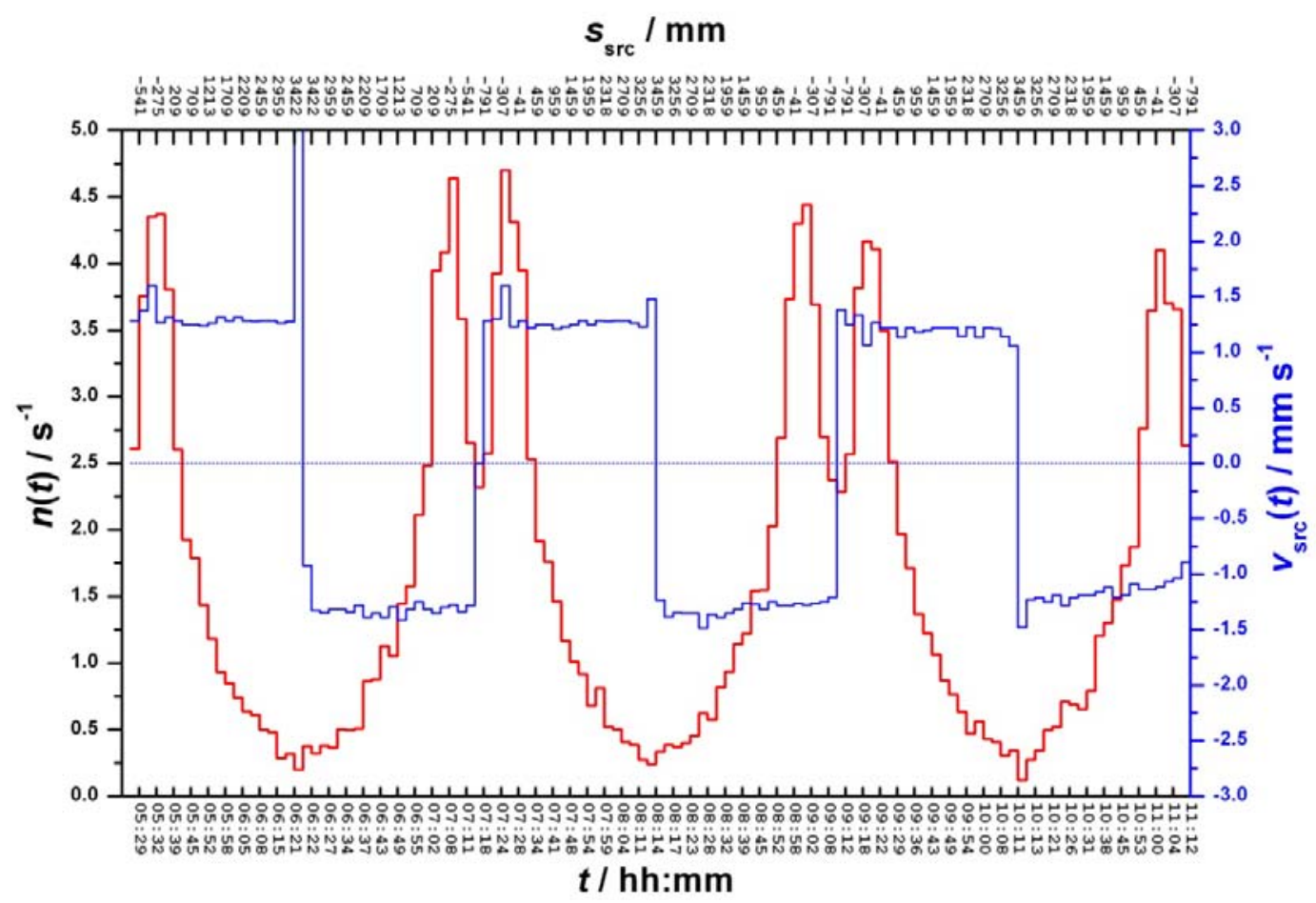

Figure 9. Measured count rates $n(t)$ as a function of time $t$ (red histogram, left ordinate) for $Z_{\text {src }}=+100 \mathrm{~mm}$. The corresponding source position $s_{\text {src }}$ is shown as upper abscissa. Please note that neither the time nor the source position is given on a continuous axis but each tag is used as bin limit. For clarity only every second value is given on the axes. The source velocity $v_{\text {src }}(t)$ is shown as blue histogram corresponding to the right ordinate. 


\subsection{Long counter data as function of path length}

For further analysis the source position $s_{\text {src }}$ on the cart track is used as reference quantity. For $Z_{\text {src }}=+100 \mathrm{~mm}$ the source was moved from $s_{1}=-936 \mathrm{~mm}$ to $s_{21}=3459 \mathrm{~mm}$. Whenever the camera showed a position tag the time was written down by hand. The positions of the tags, taking into account the offset to the source position on the cart, define the bin limits $s_{i}$ of the histogram shown in figure 10 . On the ordinate the count rates $n(s)=N_{i} /\left(s_{i}-s_{i-1}\right)$ are plotted. While the source cart moves forth and back each path length interval $s_{i}-s_{i-1}$ contains the data from several passes. Figure 10 shows the results of up to 11 measurements for one bin. The thick blue histogram is the average of each bin including the statistical uncertainty due to the total number of events counted within the bin limits.

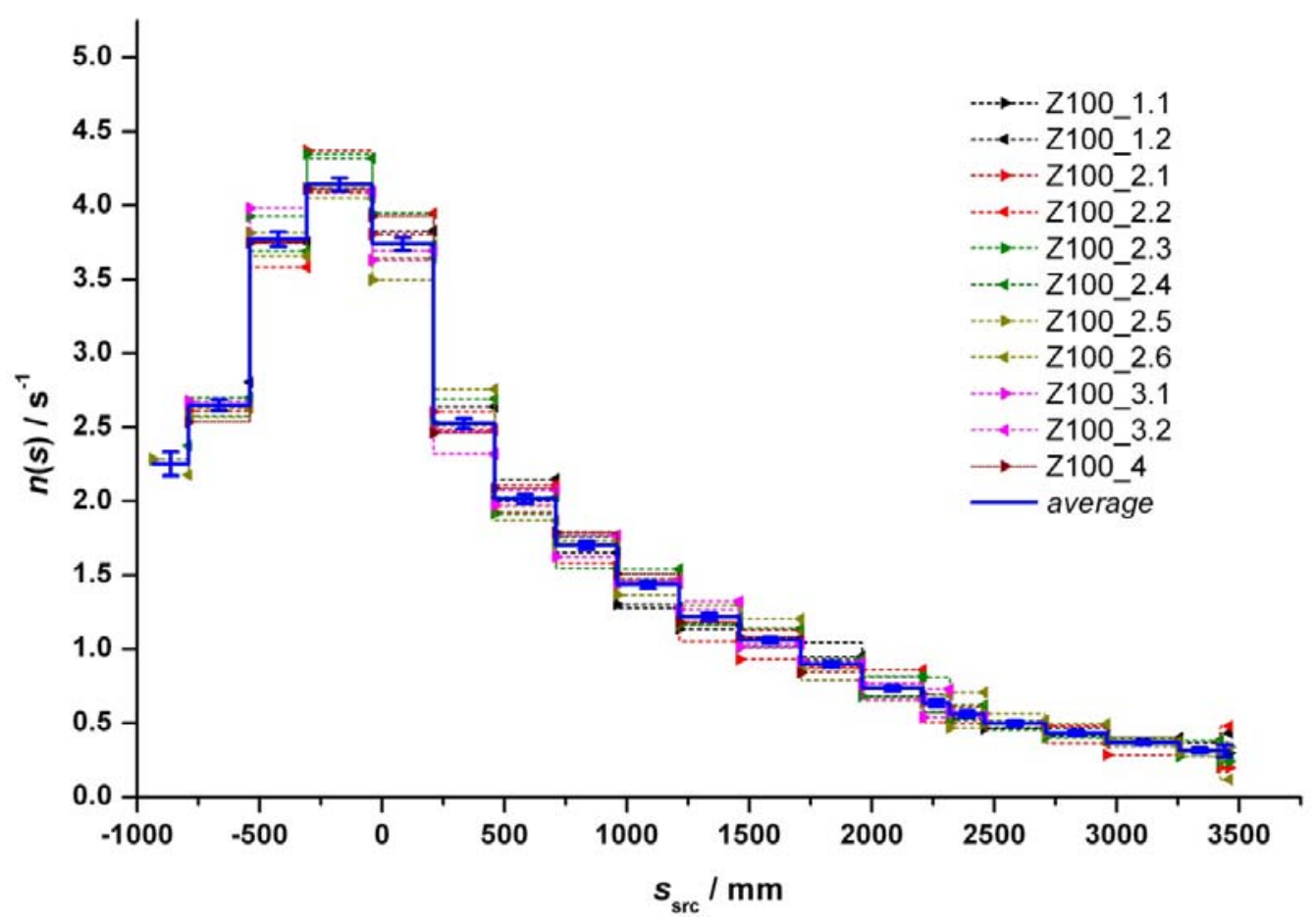

Figure 10. Measured count rates $n(s)$ as a function of source position $s_{\text {src }}$ for $Z_{\mathrm{src}}=$ $+100 \mathrm{~mm}$. The dotted lines are the results from single runs (the direction of the cart is given by the direction of the triangles). The blue histogram is the average count rate for each bin.

As already mentioned, the measurements were done for different Z-positions of the neutron source in order to get information about the possible effect on the count rates if the "source line" is expanded to an extended plasma field. The experimental results for three different heights are shown in figure 11 . The case $Z_{\mathrm{src}}=-20 \mathrm{~mm}$ was realized by removing the stand which placed the source at $Z_{\text {src }}=+100 \mathrm{~mm}$. For the measurements at $Z_{\text {src }}=+300 \mathrm{~mm}$ the neutron source was placed on another stand which had a different offset to the camera position (see sec. 5.1.3). Therefore, the bin limits of the red histogram curve for $Z_{\text {src }}=$ $+300 \mathrm{~mm}$ are shifted against the blue $\left(Z_{\mathrm{src}}=+100 \mathrm{~mm}\right)$ and the green curve $\left(Z_{\mathrm{src}}=\right.$ $-20 \mathrm{~mm}$ ). At $s=-201 \mathrm{~mm}$ and $Z_{\text {src }}=+300 \mathrm{~mm}$ the neutron source is closest to the LC with $d_{\min }(300 \mathrm{~mm})=2145 \mathrm{~mm}$. The corresponding bin with centre coordinate $s_{\mathrm{src}}=-240 \mathrm{~mm}$ 
shows a count rate of $(5.02 \pm 0.07) \mathrm{s}^{-1}$. A calculation of the count rates for the two other source heights taking into account only the increasing distance between LC and source from $2145 \mathrm{~mm}$ to $2329 \mathrm{~mm}\left(Z_{\mathrm{src}}=+100 \mathrm{~mm}\right)$ and to $2441 \mathrm{~mm}\left(Z_{\mathrm{src}}=-20 \mathrm{~mm}\right)$, respectively, give values which are only $2.7 \%$ higher than the measured values.

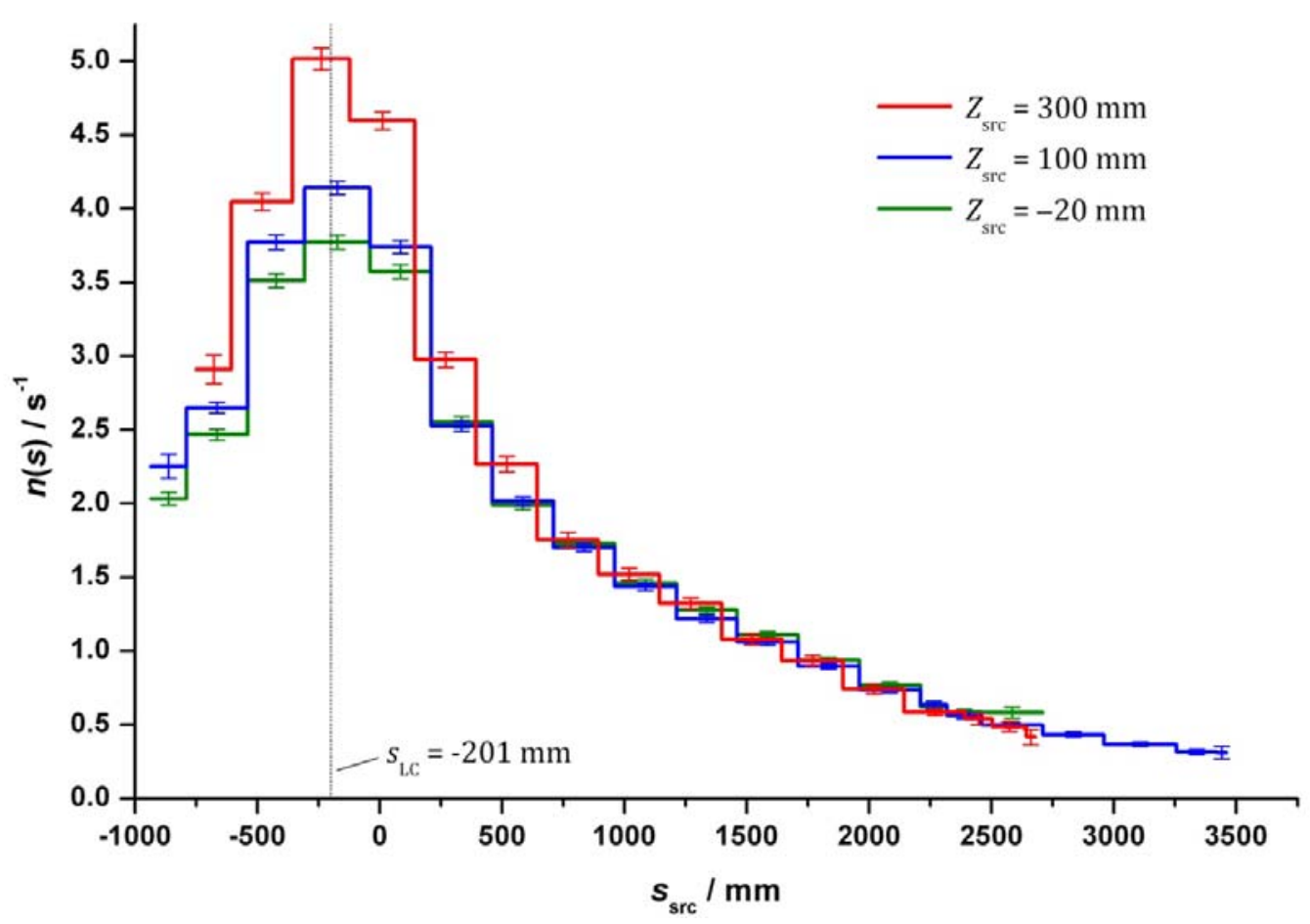

Figure 11. Measured count rates $n(s)$ as a function of source position $s_{\mathrm{src}}$ for three different source heights $Z_{\text {src }}$. The cylinder axis of the long counter crosses the source track at $s_{\mathrm{LC}}=-201 \mathrm{~mm}$ (dotted line).

In addition, figure 11 shows that the count rate becomes independent on the Z-position of the neutron source for distances larger than $75 \mathrm{~cm}$ away from the position corresponding to the closest distance between source and long counter. That means, all neutrons which start in a plane perpendicular to the plasma axis within a radius of about $15 \mathrm{~cm}$ to $20 \mathrm{~cm}$ will contribute with similar count rates to the overall result for these outer ranges of the detection cone of the long counter (see also figure 7).

\subsection{Comparison of measured data with MCNP calculations}

The analysis and interpretation of the final neutron monitor calibration before W7-X starts its operation will strongly rely on the MCNP simulation. Therefore, it was intended to reproduce the measurement with the ${ }^{241} \mathrm{AmBe}$ source in order to verify the model. For this purpose the model of the PTB Long Counter as described in section 5.1.3 was included in the Monte Carlo model for full W7-X simulation, i.e. the reaction rate of the ${ }^{10} \mathrm{~B}(\mathrm{n}, \alpha)^{7} \mathrm{Li}$ neutron detection reaction in the $\mathrm{BF}_{3}$ tube of the long counter was tallyed. The energy spectrum of the emitted neutrons was taken from ISO 8529-1 [12] and the source strength was taken as $B_{\mathrm{AmBe}}=1.49$. $10^{6} \mathrm{~s}^{-1}$. 
The modelling of the spatial distribution of the ${ }^{241} \mathrm{AmBe}$ source was done for three approaches. Firstly, the neutron source was considered as a line source along the total track of source movement and the neutron propagation on the way to the PTB LC was studied. The neutron propagation in this first case with an assumed line source is shown in figure 12 .

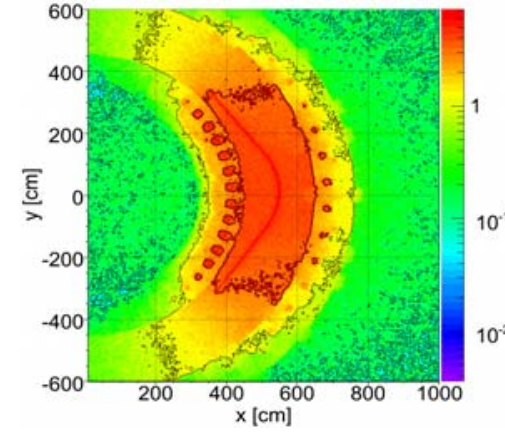

$E<600 \mathrm{meV}$

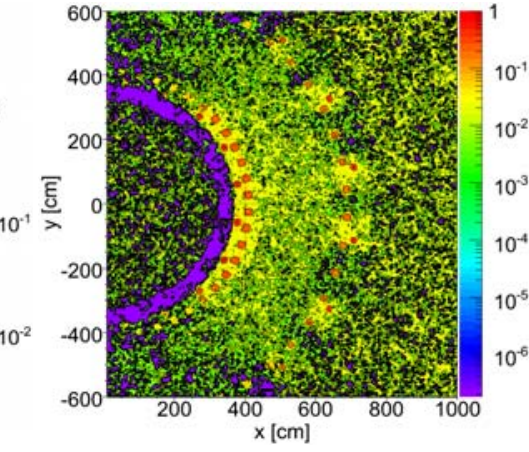

$600 \mathrm{meV} \leq E<100 \mathrm{keV}$

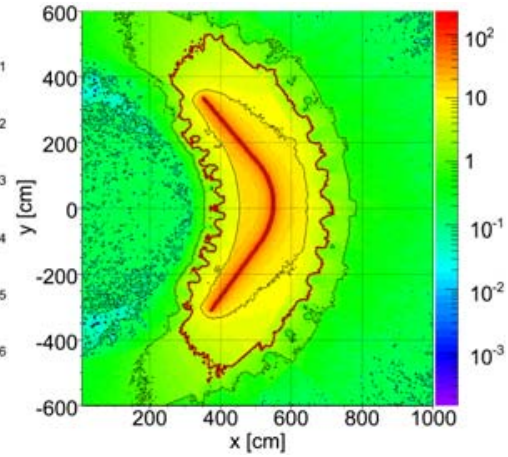

$100 \mathrm{keV} \leq E<3 \mathrm{MeV}$

Figure 12. Calculated neutron flux in $\mathrm{cm}^{-2} \mathrm{~s}^{-1}$ with starting neutrons from a line source for 3 different energy ranges.

The second approach was to consider the neutron source as a point source at fixed positions of the track ( $s$-values). In the third approach which meets the experimental conditions most suitable the source was represented by a set of line sources of approximately $25 \mathrm{~cm}$ length. For this modelling the coordinates of the cart track were used as provided by the CATIA system $\left(Z_{\mathrm{src}}=+100 \mathrm{~mm}\right)$ and the limits of the binning were taken as shown in figure 10 . The "movement" of the source was simulated by tallying the response in the $\mathrm{BF}_{3}$ tube separately for each source segment. Figure 13 shows a horizontal and a vertical cut through the MCNP model including the track system and the long counter, respectively.
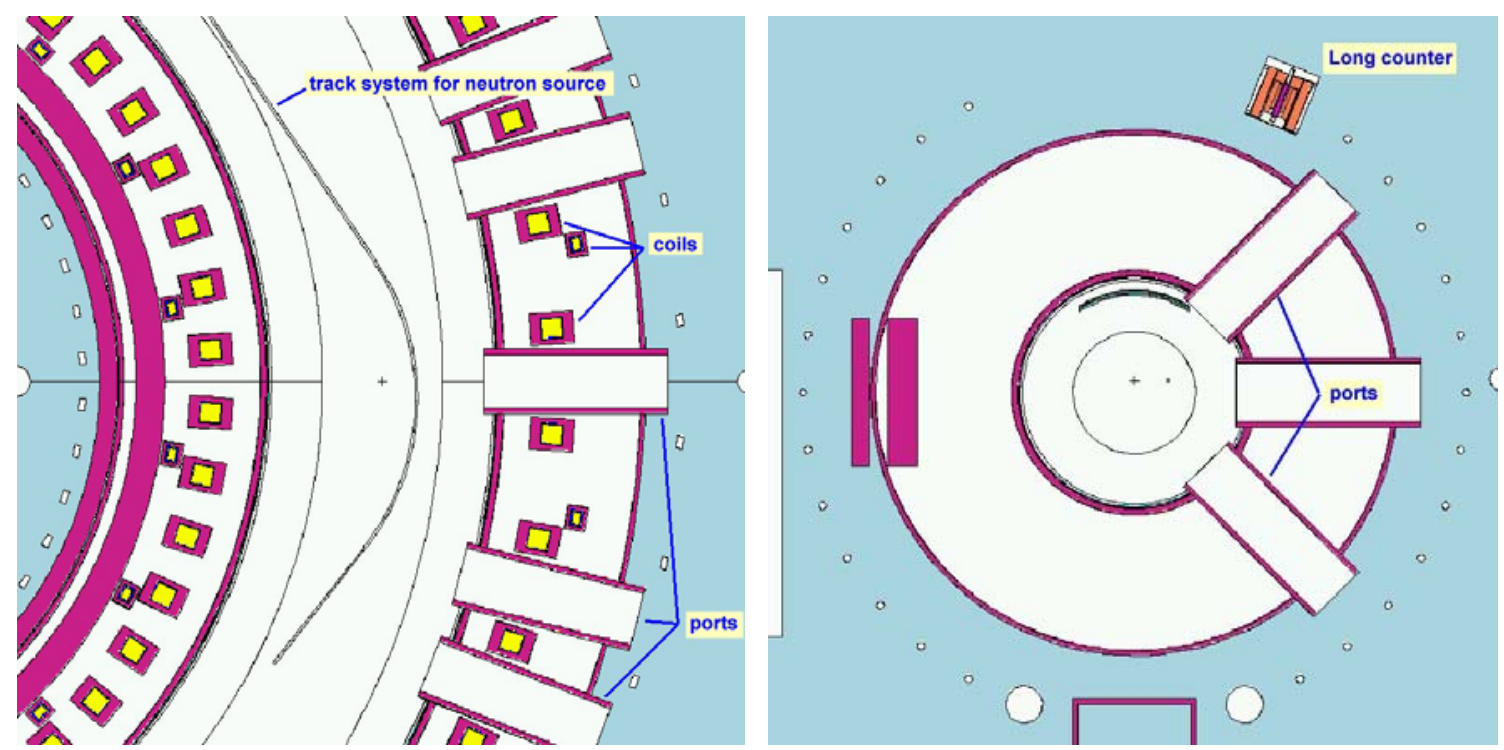

Figure 13. Left: horizontal cut through the Monte Carlo model of the plasma vessel at $Z_{\mathrm{src}}=+100 \mathrm{~mm}$. Right: vertical cut at position $s_{\mathrm{src}}=-200 \mathrm{~mm}$. 
The MCNP results of this source model were compared to the measurements performed at $Z_{\text {src }}=+100 \mathrm{~mm}$. The two histograms shown in figure 14 have been obtained without any adjustment factor. A general agreement between measurement and simulation can be noted.

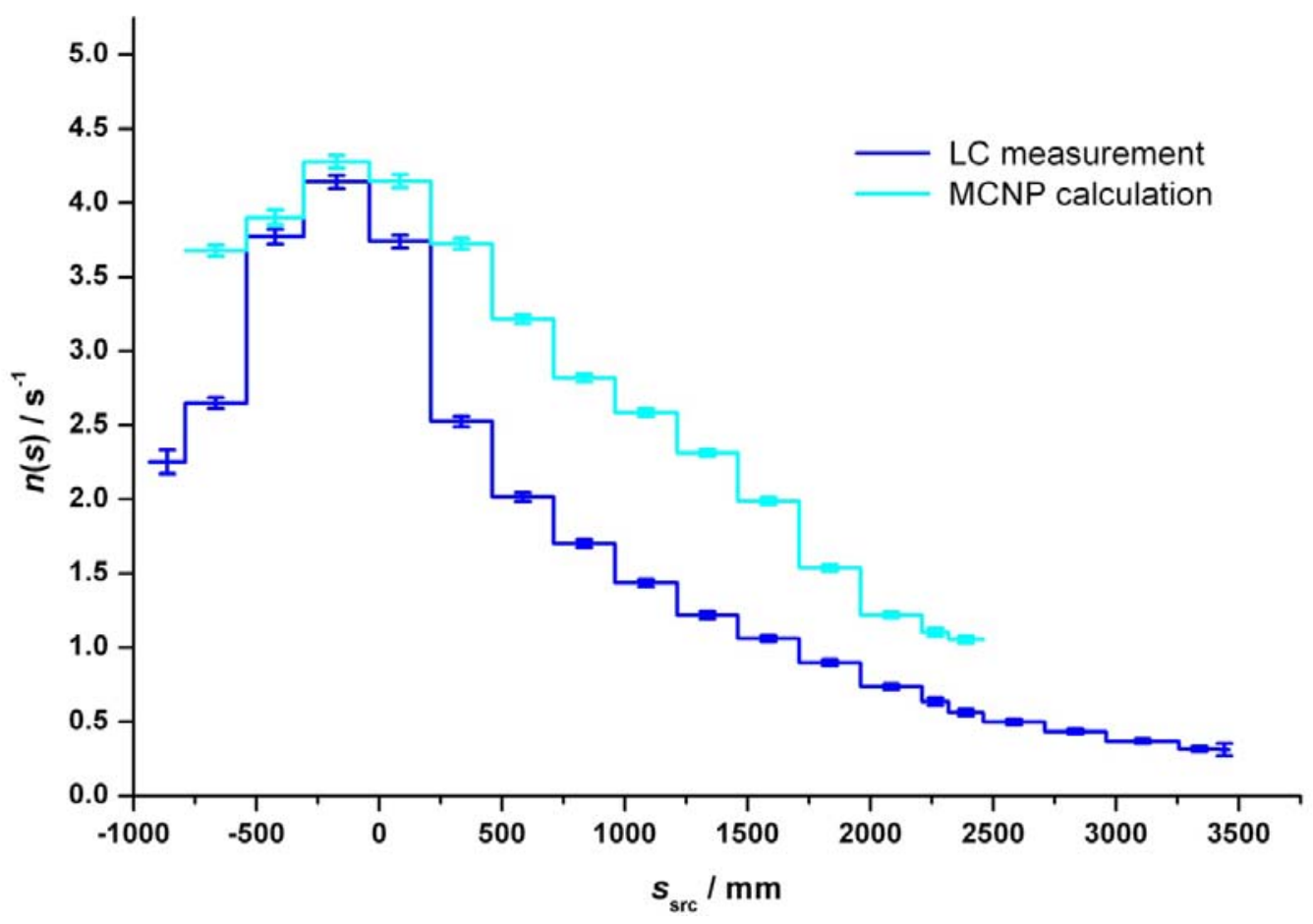

Figure 14. Count rates $n(s)$ as a function of source position $s_{\mathrm{src}}$ for $Z_{\mathrm{src}}=+100 \mathrm{~mm}$. The blue histogram is the long counter measurement and the red histogram is the result from MCNP calculations.

During real operation of the stellarator W7-X no particular spatial resolution is possible with the neutron monitors. The monitors will provide integral count rates which correspond to neutrons starting in approximately $1 / 5$ of the total plasma volume. Table 1 gives the integral count rates $n_{\text {meas }}$ and $n_{\mathrm{MCNP}}$ and their ratio for three different integration limits.

Table 1. Integral count rates $n_{\text {meas }}$ and $n_{\mathrm{MCNP}}$ for different path length limits and their ratio. The left bin edge $s_{1}$ is the same for all intervals, $\Delta s$ is the total interval length along the track line. Angle $\gamma$ is the angle between the cylinder axis of the long counter and the position of the right interval limit $s_{\mathrm{r}}$.

\begin{tabular}{rrrr|ccc}
\hline$\frac{s_{1}}{\mathrm{~mm}}$ & $\frac{s_{\mathrm{r}}}{\mathrm{mm}}$ & $\frac{\gamma}{\mathrm{deg}}$ & $\frac{\Delta s}{\mathrm{~mm}}$ & $\frac{n_{\text {meas }}}{\mathrm{s}^{-1}}$ & $\frac{n_{\mathrm{MCNP}}}{\mathrm{s}^{-1}}$ & $\frac{n_{\mathrm{MCNP}}}{n_{\text {meas }}}$ \\
\hline-307 & -41 & 3.9 & 266 & $4.14 \pm 0.04$ & $4.28 \pm 0.06$ & $1.03 \pm 0.02$ \\
-307 & 1213 & 29.0 & 1520 & $2.61 \pm 0.08$ & $3.47 \pm 0.14$ & $1.33 \pm 0.07$ \\
-307 & 2459 & 42.5 & 2766 & $1.84 \pm 0.10$ & $2.64 \pm 0.17$ & $1.44 \pm 0.12$ \\
\hline
\end{tabular}

The left integration limit is fixed to the left edge of the bin with the highest count rate $s_{1}=-307 \mathrm{~mm}$. The right limit is set to the outermost right source position in the calculation 
and the third limit is about half the distance between the outer limits. For the shortest distance of the neutron source to the long counter the agreement between measurement and calculation is excellent, $3 \%$ on an absolute scale. In this case the neutron signal is mainly influenced by the shielding of the $17 \mathrm{~mm}$ and $25 \mathrm{~mm}$ of the inner and outer vessel made of stainless steel and due to some in-scatter from materials in the vicinity below the long counter.

If an integration length of $1520 \mathrm{~mm}$ is considered with a corresponding maximum angle of sight $\gamma=29.0^{\circ}$ the calculated count rate is $33 \%$ higher than the measured value. For the maximum integration length of $2766 \mathrm{~mm}$ corresponding to an angle of sight $\gamma=42.5^{\circ}$ the overestimation of the calculation is $44 \%$.

Since this is the first attempt to compare calculations and measurements in the complex vicinity of a stellarator, the overall agreement can be considered as "good" and the ratio $n_{\mathrm{MCNP}} / n_{\text {meas }}$ could be interpreted as calibration factor. On the other hand, the difference of the shapes of the two curves in figure 14 is not yet satisfying. The number of materials and components added during the further assembling will increase the complexity of the stellarator and with it the MCNP model of W7-X. Therefore it will be an important goal of further investigations to study the line of sight from the long counter to the positions of the source within the CAD system and include more details to the MCNP model in order to better describe the shape of the measured function.

\subsection{Technical conclusions from neutron experiment at module 5}

In addition to the main aim of the neutron experiment at module 5 , several technical conclusions could be deduced with respect to an improvement of the final calibration of neutron monitors before W7-X will start plasma operation.

This test experiment has shown in general that the setup enabled an accurate measurement of neutron flux at the position of the PTB LC in dependence on the source position. But, in order to become independent of the charge capacity of accumulator packs as power supply for the movement of the neutron source cart, the final calibration is now foreseen with an external stationary power supply connected to an extra current rail parallel to the cart track.

Furthermore, it seems to be necessary to improve the smoothness of welding lines of the railway track system in order to avoid velocity changes of source movements. The third conclusion concerns the need for improving the data acquisition techniques with respect to an automatic registration of the time-space relation of the neutron source.

Finally, in addition to a measurement with an ${ }^{241} \mathrm{AmBe}$ source, a calibration with an

${ }^{241} \mathrm{AmB}$ source is foreseen. The neutron spectrum of an ${ }^{241} \mathrm{AmB}$ source is more similar to the expected DD fusion neutron spectrum what concerns mean energy and the maximum energy of emitted neutrons. This was the reason to perform this test calibration with an ${ }^{241} \mathrm{AmBe}$ source. Using two or even more neutron sources with different spectral distributions will further help to understand the differences between expected and measured calibration factors of the neutron monitors.

\section{Set of complementary neutron diagnostics at W7-X}

The neutron diagnostic tools are planned to be supplemented by a neutron activation system, a neutron profile camera, a neutron spectrometer and a $\gamma$-spectrometer to a later time.

From these complementary techniques, the neutron activation system will be realised after assembly of the neutron monitor diagnostics. This diagnostic system allows an independent 
determination of neutron fluences by means of an activation method. Hereby, thin multi-nuclide activation samples will be transported close to the plasma by means of a pneumatically based fast transport system between the diagnostic port and an analysis station outside of the torus hall. This diagnostics is foreseen for the second operational phase of W7-X with a water cooled divertor in order to stand long term and high power discharges. During the start-up phase W7-X will operate with an inertial cooled divertor. [15]

The other neutron diagnostics will then be realised together with the subsequent expansion stages of W7-X beyond the year 2018, where several diagnostics are foreseen to be added and the start-up diagnostics will be improved and extended for a higher time and spatial resolution. [2], [16]

\section{Summary}

The neutron monitor system for $\mathrm{W} 7-\mathrm{X}$ is required beside its interesting physics aspects also for official documentation of the neutron production in order to meet the annual permitted neutron budget. The central monitor above the torus will measure a spatially averaged value of the neutron flux and is optimised regarding a large opening angle of the detection range. The five outer monitors will be designed to exhibit a reduced opening angle and directional characteristics toward the plasma, even sufficient to detect neutrons over the area of one module. All six neutron monitors will be optimised such that they have a nearly constant response independent of the neutron energy.

In the test calibration experiment at module 5 of W7-X the response of a De Pangher Long Counter to a neutron source of known source strength was measured as a function of source position. The MCNP simulation of this experiment showed an overall good agreement with the measurements. The increasing difference of count rates measured with the LC with increasing distance of the neutron source is not fully understood at present. A more detailed consideration of the complex structures between plasma vessel and outer vessel including the superconducting coils will be an important task for the future in order to improve the MCNP model of W7-X. On the other hand, the neutron measurements carried out during the calibration test in module 5 of W7-X give us a valuable reference on the quality of the global MCNP model of W7-X.

\section{Acknowledgments}

The authors would like to thank A. Weller from IPP Garching for fruitful discussions and Th. Sieber from IPP Greifswald for developing the design of calibration tools for the neutron monitors. They thank further S. Dette from PTB Braunschweig for his supervision of electronics and data acquisition of the long counter during the neutron experiment.

\section{References}

[1] R. Wolf et al., From Wendelstein 7-X to a stellarator reactor, Plasma and Fusion Research, 5 (2010) 1011-1016.

[2] H. J. Hartfuß et al., Diagnostic strategy of the W7-X stellarator, Rev. Sci. Instrum. 68 (1997) 1244-1249. 
[3] B. Wolle et al., Measurements and simulations of the neutron production at W7-AS, Rev. Sci. Instrum. 70 (1999) 1197-1200.

[4] X-5 Monte Carlo Team, MCNP - A general N-Particle Transport Code, Version 5, Los Alamos National Laboratory, USA, LA-UR-03-1987 (April 2003).

[5] Y. Wu, FDS Team, CAD-based interface programs for fusion neutron transport simulation, Fusion Eng. Des. 84 (2009) 1987-1992.

[6] W. Koehldorfer, CATIA V5, Volumenmodellierung, Zeichnungen, Hanser Verlag (2009) ISBN-13: 978-3-446-41724-3.

[7] N.J. Roberts, H. Tagziria and D.J. Thomas, Determination of the effective centres of the NPL long counters, National Physical Laboratory, UK, DQL RN004 (Nov. 2004).

[8] N.J. Roberts, D.J. Thomas, V. Lacoste, R. Böttger and S. Löb, Comparison of long counter measurements of monoenergetic and radionuclide source-based neutron fluence, Radiat. Meas. 45 (2010) 1151-1153.

[9] N. J. Roberts and H. Tagziria, private communication, March 2008.

[10] F. Langner, A handy data logger for multi-purpose use, Scientific news of PTB division 6, 2007, http://www.ptb.de/en/org/6/nachrichten6/2007/61707_en.htm.

[11] L. Bertalot, S. Conroy, A. Murari, M. Reginatto, H. Schuhmacher, A. Zimbal and JETEFDA Contributors, Neutron energy measurements of trace tritium plasmas with NE213 compact spectrometer at JET, Proceedings of the $32^{\text {nd }}$ European Physical Society Plasma Physics Conference, Tarragona/Spain, 27 June - 1 July 2005, Europhysics Conference Abstracts Vol. 29C (2005), http://eps2005.ciemat.es/papers/pdf/P1_078.pdf.

[12] W. Mannhart, Evaluation of the Cf-252 Fission Neutron Spectrum between $0 \mathrm{MeV}$ and 20 $\mathrm{MeV}$, Proceedings of an IAEA Meeting, Leningrad, 1986, IAEA-TECDOC-410 (1987) 158-176.

[13] ISO Reference neutron radiations - Part 1: Characteristics and methods of production. ISO 8529-1:2001 (Geneva, Switzerland: International Standards Organization) (2001).

[14] J.W. Marsh, D.J. Thomas, M. Burke, High resolution measurements of neutron energy spectra from AmBe and AmB neutron sources, Nucl. Instrum. Methods Phys. Res. A 366 (1995) 340-348.

[15] R. König et al., Diagnostic design for steady-state operation of the W7-X stellarator, Rev. Sci. Instrum. 81 (2010) 10E133.

[16] H.J. Hartfuß et al., Diagnostics for steady state plasmas, Plasma Phys. Control. Fusion 48 (2006) R83-R150. 